\title{
Synthesis
}

\section{Integrating across Life-History Stages: Consequences of Natal Habitat Effects on Dispersal}

Michael F. Benard ${ }^{1, *, \dagger}$ and Shannon J. McCauley ${ }^{2,{ }^{*},}$

1. Department of Ecology and Evolutionary Biology, University of Michigan, Ann Arbor, Michigan 48109;

2. Center for Population Biology, University of California, Davis, California 95616

Submitted May 7, 2007; Accepted October 22, 2007;

Electronically published February 27, 2008

ABSTRACT: Ecological and evolutionary processes are affected by forces acting at both local and regional scales, yet our understanding of how these scales interact has remained limited. These processes are fundamentally linked through individuals that develop as juveniles in one environment and then either remain in the natal habitat or disperse to new environments. Empirical studies in a diverse range of organisms have demonstrated that the conditions experienced in the natal habitat can have profound effects on the adult phenotype. This environmentally induced phenotypic variation can in turn affect the probability that an individual will disperse to a new environment and the ecological and evolutionary impact of that individual in the new environment. We synthesize the literature on this process and propose a framework for exploring the linkage between local developmental environment and dispersal. We then discuss the ecological and evolutionary implications of dispersal asymmetries generated by the effects of natal habitat conditions on individual phenotypes. Our review indicates that the influence of natal habitat conditions on adult phenotypes may be a highly general mechanism affecting the flow of individuals between populations. The wealth of information already gathered on how local conditions affect adult phenotype can and should be integrated into the study of dispersal as a critical force in ecology and evolution.

Keywords: phenotypic plasticity, dispersal, metapopulation, connectivity, adaptation, gene flow.

\footnotetext{
* Both authors contributed equally to this work.

† E-mail: mfbenard@umich.edu.

‡ E-mail: sjmccauley@ucdavis.edu.

Am. Nat. 2008. Vol. 171, pp. 553-567. (c) 2008 by The University of Chicago. 0003-0147/2008/17105-42606\$15.00. All rights reserved.

DOI: $10.1086 / 587072$
}

How processes that occur on local scales (e.g., local recruitment or evolutionary response to natural selection) interact with one another through dispersal across a regional scale has important implications for the persistence of species (Hanski and Gilpin 1997; Hanski 1999), evolutionary diversification (Smith et al. 1997; Nosil and Crespi 2004), and the structuring of ecological communities (Travis 1996; Holyoak et al. 2005; Urban and Skelly 2006). One fundamental way these processes are linked is through individuals that develop as juveniles in one environment and then, as adults, either disperse to new environments or remain in their natal environment. By altering the traits or quantity of dispersers between different environments, phenotypic plasticity induced by natal habitat conditions can have profound consequences for metapopulation and metacommunity dynamics as well as genetic differentiation and adaptation of populations. These effects include strongly reducing the ability of metapopulations to persist (Vuilleumier and Possingham 2006), skewing estimates of connectivity between populations, and creating unidirectional gene flow and thus asymmetries in the geographic patterns of local adaptation and maladaptation (Stanton et al. 1997; Kawecki and Holt 2002). While natal habitat-induced plasticity of adult phenotypes has received considerable attention by ecologists working at local scales (e.g., Roach and Wulff 1987; Pechenik 2006), how those developmental environments may affect the quality and quantity of dispersing individuals has received very little attention (Ronce et al. 2001; Clobert et al. 2004). This is an important gap in our understanding of ecological and evolutionary processes. Carryover effects between the conditions in which an individual develops and dispersal performance and behavior are likely to be a highly general mechanism that creates dispersal asymmetries between habitat patches that vary in condition. Thus, this form of carryover effect generates an intrinsic link between the local developmental environment and the regional connectivity of populations. Understanding these linkages provides a potentially powerful tool for relating 
local and regional scale processes through the traits of individuals.

Our understanding of the relationship between an individual's phenotype at the dispersal stage and its propensity to disperse remains tenuous for many systems. Few studies in any system have fully explored how intraspecific phenotypic variation affects dispersal. Regardless of study system, we also lack a clear understanding of how the distribution of dispersal-related phenotypes is affected by distinct developmental conditions that vary between habitat patches and the resulting consistent dispersal asymmetries between habitats with different environmental characteristics. Our goal here is to develop a conceptual framework (fig. 1) for investigating how dispersal asymmetries may arise between patches as a result of different local environments that each alter individual development in a distinct way and thus have different carryover effects on adult dispersal. We propose that the ecological and evolutionary interactions between patches in a set of populations must take into account how environmental conditions affect the number and phenotypes of dispersing individuals. This is best investigated in a holistic manner, in which all stages leading from individual developmental environments to the ecological and evolutionary consequences of environmental variation among patches are considered. Specifically, it requires biologists to evaluate the spatial structure of environmental variation affecting juvenile development and thus adult dispersal phenotypes (fig. 1A). Then, the consequences of these developmental conditions for the behavioral, morphological, and physiological phenotypes that affect dispersal must be identified (fig. 1B). Finally, how differences in the relative numbers and quality of dispersers moving into new habitats affect metapopulation persistence, metacommunity structure, and local adaptation need to be considered (fig. 1C).

In this article, we will discuss the consequences of natal habitat effects on disperser phenotypes. We define the term "natal habitat" broadly; it is the environment an individual experiences early in its life that may affect its phenotype at the dispersal stage. The specific life stage in which an individual occupies its natal habitat will vary among taxa. For instance, animals with complex life cycles would inhabit the natal habitat during the larval stage; the phenotype at metamorphosis may affect the dispersal of juveniles that ultimately breed in an adult habitat. Similarly, the conditions (e.g., soil type, shade) in which a plant develops are the natal habitat for that plant's seeds. Those seeds are then dispersed at a rate that may be affected by conditions experienced by the maternal parent.

In some cases, the effects of natal habitat on disperser phenotypes are obvious. For example, reviews from diverse systems conclude that many animals with complex life cycles exhibit adaptive phenotypic plasticity in the larval
Natal Environment
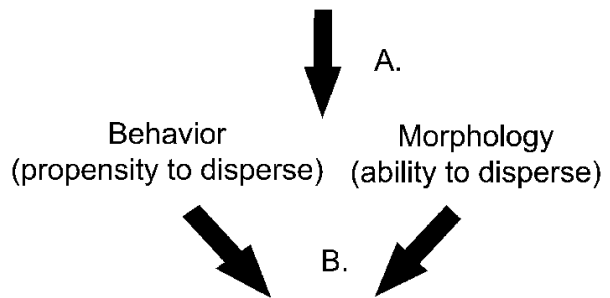

Fraction and phenotype of individuals that emigrate from a patch

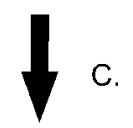

Ecological and Evolutionary consequences of asymmetries in disperser number, dispersal distance, and the phenotypic composition of the disperser pool

Figure 1: Conceptual view of the path from natal habitat effects on individuals to ecological and evolutionary consequences on populations and communities. A distinct series of research questions arises at each step along this path. A, Why do natal conditions create specific adult phenotypes? For example, are adult phenotypes a by-product of adaptation to the juvenile environment or an adaptation to disperse? $B$, How does natal habitat-induced variation in adult phenotype affect dispersal? For example, are more individuals likely to disperse or disperse longer distances? Will immigrants into a new habitat have a different phenotype than the residents that they interact with? $C$, What are the consequences for ecological and evolutionary processes? For example, does altered dispersal affect our estimate of connectivity? Does environmentally induced asymmetry in dispersal increase extinction risk? Does environmentally induced asymmetry in dispersal cause an asymmetry in local adaptation?

phenotype that can have carryover effects into the postmetamorphic stage (Benard 2004; Pechenik 2006). The effects of natal habitat, however, may also be subtle; we include maternal effects in our definition. For instance, stress experienced by female vertebrates can affect the phenotype of their offspring (birds: Hayward and Wingfield 2004; mammals: Marchlewska-Koj et al. 2003; lizards: Meylan and Clobert 2004). Maternal effects in plants can affect seed quality and ability to disperse (Roach and Wulff 1987; Imbert and Ronce 2001). We chose a broad definition of natal habitat effects because the ultimate ecological and evolutionary consequences are similar, regardless of whether the natal habitat effect is a specific type of larval host that causes insects to disperse longer distances or a maternal effect influenced by habitat conditions in a plant that causes seeds to develop structures to disperse farther. Also for clarity, we have restricted ourselves to a discussion of natal dispersal, the movement of an individual from its natal site before first reproduction (Clobert et al. 2001). Throughout our article, we use the general term "dispersal" to refer to natal dispersal. When migra- 
Table 1: Predictions for how natal habitat-induced phenotypic plasticity may affect the probability that an individual disperses

\begin{tabular}{|c|c|c|c|}
\hline $\begin{array}{l}\text { Dispersal condition and natal } \\
\text { habitat effects }\end{array}$ & Nature of effect & Example & $\begin{array}{c}\text { Effect on dispersal } \\
\text { probability }\end{array}$ \\
\hline \multicolumn{4}{|l|}{ Adaptive: } \\
\hline Relative cost of dispersal & Increase & $\begin{array}{l}\text { Natal habitat causes individuals to be smaller } \\
\text { and thus more susceptible to dispersal } \\
\text { mortality }\end{array}$ & Decrease \\
\hline Mating success in new habitat & Increase & $\begin{array}{l}\text { In social (lekking or chorusing) breeding sys- } \\
\text { tems, males that develop at low density and } \\
\text { are larger may move to higher-density habi- } \\
\text { tats because they are able to establish territo- } \\
\text { ries and attract females; higher male densities } \\
\text { attract more females }\end{array}$ & Increase \\
\hline Performance of offspring & Increase & $\begin{array}{l}\text { Larger seeds have greater competitive ability in } \\
\text { new habitat }\end{array}$ & Increase \\
\hline \multicolumn{4}{|l|}{ Nonadaptive: } \\
\hline Dispersal capacity & Increase & Lower wing loading in insects & Increase \\
\hline Dispersal period length & Increase & $\begin{array}{l}\text { Smaller amphibian metamorphs take longer to } \\
\text { reach maturity }\end{array}$ & Increase \\
\hline
\end{tabular}

Note: Predictions depend on whether dispersal is a direct adaptation itself or a by-product of other activities.

tion or breeding dispersal are discussed, we distinguish these as such.

\section{How Does Natal Habitat Affect Dispersal?}

Numerous models have been put forward to provide an adaptive explanation for why dispersal occurs. These can be grouped into three general categories: (1) hedging against environmental variation, (2) avoiding kin competition, and (3) avoiding inbreeding (all three are reviewed in Johnson and Gaines 1990; Clobert et al. 2001). A common theme in most of these models is that there is a fitness cost associated with dispersal. Natal habitat effects on disperser phenotypes may affect the magnitude of those costs, thereby generating an asymmetry in the relative costs of dispersal and thus affecting the conditions under which dispersal is adaptive. For example, in the absence of competition with kin or the risk of inbreeding, we would predict individuals to disperse from their natal habitat when their fitness if they disperse is greater than their fitness if they remain in the natal habitat (Johnson and Gaines 1990; Bélichon et al. 1996). The fitness of a dispersing individual is a function of the costs it incurs while dispersing, its mating success in the new habitat, and the reproductive value of its offspring to the dispersal phase. In contrast, the fitness of a resident individual is a function of its mating success in the new habitat and the reproductive value of its offspring. Both the costs incurred during dispersal and the mating success of individuals in their adult habitat are partially a function of an individual's phenotype at the dispersal stage. The phenotype of dispersers is itself a function of the genotype by environment interaction of individuals developing in a specific natal habitat. By affecting an individual's phenotype at the dispersal stage, natal habitat-induced phenotypic plasticity can alter the ratio of fitness costs to fitness benefits of dispersing and, thus, whether the decision to disperse is adaptive (table 1). While such natal habitat effects on the costs of dispersal have been identified in a few systems, no theoretical work has included them. We consider how natal habitat conditions affect an individual's phenotype at the dispersal stage and how this may in turn alter the cost-benefit structure of the decision to disperse.

Alternatively, dispersal itself may not be an adaptation to the natal environment and local landscape. Instead, dispersal may be a function of movement in organisms that utilize different environments during the natal habitat and prereproductive phases. For example, in amphibians with biphasic life histories, the dispersal period between metamorphosis and reproduction may account for most of the organism's growth and life span (e.g., 2-month larval period vs. 2-year terrestrial juvenile stage). During this time, individuals may move over the terrestrial landscape, seeking food and suitable terrestrial habitat until they reach sexual maturity. Thus, the probability that an individual disperses may be a function of its movement capacity and the duration of time that it spends between metamorphosis and the dispersal phase, which in turn may be a function of natal habitat effects (table 1). Under this scenario, natal habitat effects on the phenotype of dispersers are not an adaptation to disperse; rather, they arise through a correlation between a phenotype (e.g., size) induced by development in the natal habitat and an individual's probability of dispersal. 
Natal conditions can affect the probability that an individual will leave the natal habitat patch before breeding in two distinct ways: (1) by affecting the capacity of an individual to disperse and (2) by affecting the motivation of an individual to disperse. Both effects on dispersal are quantifiable and may also interact with one another. These effects may be a direct adaptation, for example, if an individual develops a dispersal-specific phenotype to escape a low-quality environment. Alternatively, natal habitat effects on dispersal capacity and propensity may be due to environmental constraints; for example, dispersing from a poor-quality environment may be adaptive, but low resources in the natal environment prevent an individual from developing a dispersal-specific phenotype. Finally, these effects may be due to correlations among phenotypes developed as an adaptation to the natal environment. For example, low growth rates in the natal environment may be an adaptation to reducing predation risk (e.g., Benard 2004). The resulting smaller size at the dispersal stage may affect dispersal propensity or capacity but is not in itself an adaptation that optimizes an individual's dispersal strategy. By altering the phenotypes of individuals that do disperse, natal habitat effects may change the nature of the ecological interactions between dispersers that enter a new population and the residents that they encounter there. Here we discuss examples of these phenomena to demonstrate that they are likely to be common. Later, we provide examples that show how natal habitat effects may alter ecological and evolutionary processes.

\section{Dispersal Capacity}

One of the clearest patterns relating dispersal ability and local habitat conditions is found in insects with wing dimorphisms. These species possess dispersal polymorphisms that result in distinct flight-capable and flight-incapable morphs. These dispersal polymorphisms include both morphological (e.g., larger wings) and physiological (e.g., increase in production of triglycerides) changes (Zera and Denno 1997). Reviews of dispersal polymorphisms (Dingle 1996; Zera and Denno 1997) conclude that the developmental switch to flight-capable morphs is most commonly associated with poor or deteriorating local environments, including temperature and photoperiod changes associated with seasonal climatic change as well as crowding and low food quality. Exposure to predators (Weisser et al. 1999) and parasitoids (Sloggett and Weisser 2002) also induces increased production of flight-capable winged offspring in pea aphids (Acyrthosiphon pisum). Polymorphic flight strategies in these species appear to be favored by costs associated with the production and maintenance of wings and/or flight musculature, which are high enough to impose trade-offs between dispersal and fecun- dity (Roff and Fairbairn 1991). In species of plant hoppers, differences in the proportion of the population that develops the winged form can exist at both large and small geographic scales (Denno et al. 2001). Thus, there exists spatial variation in these habitat conditions that has the potential to generate dispersal asymmetries between patches.

Discrete phenotypes that affect the capacity of individuals to disperse are not limited to insects with wing polymorphisms. For example, many species of plants exhibit seed heteromorphisms affecting seed dispersal, such as the presence versus absence of wings (Imbert and Ronce 2001). At least some of these seed heteromorphisms are induced by the environment experienced by the mother (Imbert and Ronce 2001). Another discrete polymorphism that can affect dispersal is facultative paedomorphosis in salamanders. For some species of salamanders, the quality of the pond (e.g., resource availability) in which the larvae develop determines whether they develop into either a terrestrial adult capable of dispersing overland to a new pond or a completely aquatic gilled adult restricted to its natal pond (Whiteman 1994). Thus, there are many examples of organisms with discrete environmentally induced phenotypes that may affect dispersal capacity.

Many other taxa exhibit continuous, rather than dichotomous, variation in natal habitat-induced phenotypes that has carryover effects into later life-history stages (Dufty et al. 2002; Benard 2004; Pechenik 2006). Although clearly not all traits affected by carryover effects from the developmental environment to the adult phenotype will impact the dispersal ability of individuals, natal conditions often impact major morphological and physiological parameters, such as body size, that have the potential to affect dispersal. Distinguishing the impact of local developmental conditions on dispersal probability in animals without distinct and dichotomous dispersal morphs requires identification of (1) how developmental conditions affect phenotype at the dispersal stage and (2) how that phenotype affects dispersal probability (fig. 1).

One common way in which natal conditions are likely to affect dispersal is through their influence on an animal's size and the subsequent effects of body size on dispersal ability. For example, interspecific comparisons indicate that body size is positively correlated with dispersal in both mammals (Sutherland et al. 2000) and birds (Paradis et al. 1998). In mammals, one of the classic studies on proximal factors associated with dispersal followed individually marked Belding's ground squirrels (Spermophilus beldingi) in two populations (Holekamp 1984, 1986). Males are the most frequent dispersers, and in both populations, body size was positively associated with dispersal. Attaining a threshold body size was critical as a proximate mechanism triggering dispersal. Although body size was associated 
with the initiation of male dispersal in both populations, these sites differed in juvenile body size. The site with higher population density had smaller juveniles that dispersed shorter distances than individuals from the lowerdensity site, suggesting that differences in natal conditions acting on body size also influenced dispersal behavior. Intraspecific comparisons in birds have also found body size to be positively associated with the timing (Lens and Dhondt 1994), distance (Hockey et al. 2003), and rates (van der Jeugd 2001) of natal dispersal. In some cases, there is a clear link to habitat condition. For example, in crested tits (Parus cristatus), individuals from lower-quality habitats (isolated forest plots) were slower to reach a threshold size and disperse than individuals from higherquality intact forests (Lens and Dhondt 1994). In other cases, phenotypic variation within a single breeding colony resulted in differences in body size and dispersal behavior (van der Jeugd 2001). This demonstrates that the effects of body size, determined during development, on dispersal can arise both between and within populations.

Similarly, laboratory studies demonstrate that larval conditions affect the size of metamorphosing frogs and that these larval conditions indirectly affect postmetamorphic dispersal capacity; larger frogs will hop for a greater distance before tiring or will hop greater distances in a single jump (e.g., Beck and Congdon 2000). Phenotypic plasticity in amphibian size at metamorphosis is found in response to many different forms of environmental variation (e.g., resource availability: Morey and Reznick 2000; predation risk: Benard 2004). However, the link between amphibian movement capacity and actual dispersal in nature has received little empirical attention.

In insects, larval environment affects adult body size (competition: Rossi et al. 1996; resource quality: Blanckenhorn 2006; predation risk: Benard 2004), and this may translate into differences in dispersal capacity between populations that differ in larval environmental conditions. Interspecific comparisons of dispersal behavior in flying insects frequently indicate that larger species have greater dispersal capacities (Nieminen et al. 1999; Roslin 2000; Angelibert and Giani 2003) and are more likely to be migratory (Roff 1991). Body size can affect the ability to maintain directed flight at the speeds necessary to overcome ambient airspeeds, so that flight remains directed rather than a passive function of air currents (Dudley 2000). In odonates, the mass of the thoracic musculature is a strong predictor of power output (Schilder and Marden 2004), suggesting that aspects of body size may have a direct functional role in dispersal ability in this group and in other winged insects where the thorax houses the musculature that powers flight. Intraspecific comparisons of body size and dispersal rate in odonates have found a positive relationship between size and dispersal (Anholt
1990; Michiels and Dhondt 1991; Conrad et al. 2002). Anholt's study is of particular interest because he experimentally manipulated the larval environment of a damselfly (Enallagma boreale) and then evaluated the consequences of developmental environment on both body size at metamorphosis and dispersal probability. Although the number of dispersal events observed was relatively low, individuals that dispersed were significantly heavier at emergence than individuals that did not disperse (Anholt 1990). This study demonstrated a linkage between developmental conditions, adult phenotype, and dispersal behavior. Anholt's results also indicate that high-quality habitats produced larger individuals with greater dispersal abilities, a potentially common form of the habitat condition-dispersal ability relationship. This suggests that high-quality habitats may contribute a greater proportion of dispersers to the regional pool. Although the "habitat conditions" were experimental treatments, the differences between these treatments parallel those observed in natural environments, demonstrating that habitat conditions can produce asymmetric dispersal patterns between natural habitats that differ in larval resource availability.

While body size may be important in affecting dispersal ability in many taxa, other aspects of the individual's morphological or physiological phenotype will also influence dispersal ability and may even interact with body size to determine dispersal ability. These too can be affected by natal habitat conditions. For example, Merckx and Van Dyck (2006) reared caterpillars of speckled wood butterflies (Pararge aegeria) in low-quality (i.e., agricultural) and high-quality (i.e., woodland and woodland fragments) sites. These rearing environments predictably affected the flight morphology of the adult butterflies. Individuals that developed in the low-quality site had higher wing loadings, a trait positively associated with flight speed and performance (Dudley 1990; Srygley and Chai 1990), than individuals that developed in the high-quality site. Thorax mass and wing loading strongly affect flight capacity in Lepidoptera (Srygley and Chai 1990). In a study of multiple species of Neotropical Lepidoptera, Srygley and Chai (1990) found that populations located in pasture habitats dispersed more and differed in wing and thorax allocation patterns, corrected for body size. Individuals from pasture habitats had longer and wider forewings and heavier thoraxes than populations in forested landscapes, differences the authors attributed to phenotypic plasticity. Larger wings relative to body size decrease wing loading, while a heavier thorax is associated with increased flight power in some flying insects (Srygley and Chai 1990; Schilder and Marden 2004). Dispersal is a potentially costly process; body size, body condition, and patterns of morphological allocation are all likely to affect the ability of individuals to undertake and accomplish dispersal. For individuals 
engaged in natal dispersal, these traits have been shaped in part by the natal habitat conditions they have encountered. Thus, local environment-induced differences in dispersal ability between individuals emerging from alternative habitats potentially create asymmetries in dispersal frequency from sites and can change mean dispersal distances of individuals from different habitats.

\section{Dispersal Propensity}

In animals with active dispersal where there is strong behavioral control over dispersal, motivation can control dispersal rate. For example, in insects with wing dimorphisms, not all dispersal-capable individuals will leave their natal habitat; winged individuals are only "potential" dispersers (Roff and Fairbairn 2001). Therefore, motivation to disperse can be the critical determinant of whether dispersal actually occurs; that is, the potential disperser becomes an actual one. Motivation to disperse can be viewed on two levels: the proximate external or internal cues that induce dispersal and the ultimate evolutionary history that has led to a specific cue triggering dispersal. Identification of the ultimate explanations for why individuals disperse is an active area of research and has been recently reviewed (Clobert et al. 2001; Kisdi 2002). A number of factors may select for dispersal, including avoiding negative interactions with kin such as competition (Hamilton and May 1977; Comins et al. 1980) and inbreeding (Bengsston 1978; Crespi and Taylor 1990). Selection may also favor the evolution of dispersal in patchy environments where there is temporal fluctuation in habitat quality that is at least partially uncorrelated between patches (Levin et al. 1984; McPeek and Holt 1992), including variation in population densities (Holt and McPeek 1996; Doebeli and Ruxton 1997). Under these conditions, dispersal can spread the risk associated with local population extinctions, and dispersing individuals have the potential to increase their fitness by dispersing to higher-quality environments. These two processes can maintain divergent (i.e., low and high) dispersal strategies (Mathias et al. 2001; Kisdi 2002) as well as conditional dispersal strategies (McPeek and Holt 1992) within a population. Although dispersal can provide a number of potential fitness benefits, the costs of dispersal, including increased risk of mortality (Van Vuren and Armitage 1994; Baker and Rao 2004) and reduced resources available for reproduction (Cohen and Motro 1989; Roff and Fairbairn 1991; Zera and Denno 1997), can be significant and decrease the fitness value of dispersal, even from relatively poor environments. If an individual's probability of dispersing is adaptive, then an individual's dispersal propensity will be an evolutionary response to the relative costs and benefits of dispersal for that genotype. By affecting an individual's phenotype, natal habitat- induced plasticity in disperser phenotype can affect the behavioral propensity to disperse by influencing the cost of dispersal, the mating success of an individual in a novel habitat, and potentially even the performance of offspring in a new habitat.

Natal habitat effects can not only influence the propensity for an individual to leave a habitat and disperse but also alter the type of habitat that individuals prefer or are able to settle in. Development in one habitat may cause an individual to prefer to settle in that habitat type over other habitats. While reviews of studies of some taxa have argued that there is little support for this phenomenon, (e.g., arthropods: Jaenike 1990), more recent work has suggested that this habitat preference induction may actually be taxonomically widespread (Davis and Stamps 2004). Natal habitat effects are not limited to individual habitat choices; for instance, weaker birds may be forced to settle in suboptimal habitats (Garant et al. 2005). Thus, natal habitat conditions can affect all phases of dispersal: the probability that an individual leaves, how far it travels during dispersal, and what habitat it ultimately settles in.

The proximate cues that trigger dispersal away from the natal habitat may be internal and specific to an individual's phenotype. For example, body condition appears to be a critical proximate factor affecting both the decision to disperse and its timing. In some mammals, body composition and, particularly, the level of fat stores are critical in triggering dispersal, with higher fat stores being associated with increased motivation to disperse (O'Riain et al. 1996; Nunes et al. 1998). The relationship between body size and dispersal may be affected by the social context in which dispersal decisions are made by individuals (Hanski 1991), suggesting that body size may affect both ability and motivation to disperse. Thus, natal habitat can alter motivation to disperse simply by affecting the body condition of individuals when they reach the dispersal stage.

Proximate cues that trigger dispersal may also be a function of the natal environmental conditions that an animal experiences. For example, juvenile common lizards ( $\mathrm{La}$ certa vivipara) whose mothers were reared in food-limited environments were less likely to disperse than common lizards whose mothers came from food-rich environments (Massot and Clobert 1995). The effect of maternal food limitation on juvenile dispersal appears to be mediated in part by the vertebrate stress hormone corticosterone; experimental application of corticosterone to gravid female common lizards caused their offspring to be more likely to disperse (de Fraipont et al. 2000; Vercken et al. 2007). Further, the effects of corticosterone on offspring dispersal can be modified by maternal condition; corticosterone addition decreased dispersal rates in offspring of larger females (Meylan et al. 2002, 2004). These results suggest that multiple maternal signals of stressful or poor con- 
ditions may increase offspring dispersal propensity. Individuals from poor or deteriorating habitats may develop a high motivation to disperse and leave these conditions (McPeek and Holt 1992; Dingle 1996; Coll and Yuval 2004). In contrast, motivation to disperse may also be related to the probability of dispersal being successful (Bonte et al. 2003), and very high-quality habitats may produce more individuals capable of successfully dispersing to new habitats than are produced by low-quality habitats. Currently, we lack theory on how natal habitat effects on individual characteristics such as body size affect the evolution of conditional strategies. However, the empirical examples discussed above suggest that selection may act to increase the motivation to disperse at the extreme ends (low and high) of the habitat quality spectrum. For individuals coming from low-quality habitats, the fitness costs of remaining in these habitats to breed may be high relative to the costs of dispersal, even if dispersal costs for these individuals are quite high. Alternatively, if individuals coming from high-quality habitats are physically more capable of dispersing successfully, the costs of dispersal may be greatly reduced relative to potential benefits. This suggests that for any system, it is critical to evaluate the role of natal habitat in affecting motivation to disperse and how selection for this behavior may change across a gradient of habitat quality.

\section{Interactions between Dispersal Ability and Propensity}

Simply because an organism has the capacity to disperse does not mean that it will disperse. For example, if we compare populations in "high-quality" and "low-quality" habitats (defined with respect to individual performance), the individuals developing in the high-quality habitat may have a greater capacity to disperse (e.g., if they are larger). Yet if the costs of dispersal (e.g., mortality risk or energetic costs) are high, the individuals from the high-quality habitat may have evolved a low motivation to disperse. In contrast, individuals from low-quality habitats may be able to accrue greater benefits by dispersing away from the lowquality habitat, despite their reduced dispersal capacity. Similar effects are likely to occur within populations. For example, the effects of body size on dispersal in mammals may shift on the basis of social and environmental conditions. A study of dispersal in the common shrew (Sorex araneus; Hanski 1991) found that when shrew and microtine rodent densities and dispersal rates were low, dispersers were smaller than residents and were probably social subordinates. When shrew and microtine densities were high, dispersal rates increased, and dispersers were larger in some morphological measures. Although the consequences of body size for dispersal shifted between these periods, there was consistently a size bias in the individuals that dispersed.

Within species, there are often genetically based tradeoffs such that an individual that has high fitness in one environment has low fitness in a second environment. Within a population, genotypes adapted to a specific natal condition may exhibit a different phenotype at the dispersal stage as well as a different propensity to disperse than an individual with a genotype that is not adapted to the specific local condition. In the side-blotched lizard (Uta stansburiana), males exhibit three distinct behavioral phenotypes, and the social environment strongly affects the fitness of each behavioral phenotype (Sinervo et al. 2006). The propensity of hatchlings to disperse is determined in part by an interaction between hatchling condition (i.e., a maternal effect) and sire genotype (Sinervo et al. 2006). The interaction between an individual's genetic potential for success in a habitat and its potential to disperse is likely to be a general phenomenon.

Interactions between natal habitat effects, phenotype, and dispersal have been described in other systems as well. For example, in great tits (Parus major), individuals born in low-quality habitats that develop high-quality phenotypes (higher nestling mass) are more likely to disperse into and breed in higher-quality habitats than individuals from low-quality habitats with low-quality phenotypes (Verhulst et al. 1997). Cross-fostering experiments in this system found that nestling mass was principally determined by environmental conditions (Shapiro et al. 2006). This suggests that the natal environment can affect the ability to successfully disperse to a new habitat, including the ability to establish territory in the new environment, and this interacts with an individual's propensity to do so. Similar patterns were found in female black-capped chickadees (Parus atricapillus), where better natal nutrition was associated with movement into and settlement in highquality habitats (van Oort and Otter 2005), and flamingoes (Phoenicopterus ruber roseus), where higher-quality natal habitats were positively associated with both body condition and dispersal (Barbraud et al. 2003). Another example of this phenomenon can be found in insects with larvae that feed on different host plants. Coll and Yuval (2004) experimentally investigated dispersal morphology and propensity in potato tuberworms (Phthorimaea operculella) reared on both a high-quality (potato) and a lowquality (tomato) host. The adult moths were much more likely to fly from the low-quality host than from the highquality host. Further, on the low-quality host, the individuals that were more likely to fly were those with morphology more appropriate to dispersal: they had lower wing loadings. No such interaction occurred on the highquality host plant. There is a high potential for interactions between dispersal ability and propensity to generate non- 
linear relationships between habitat condition and net dispersal rates. Distinguishing the effects of dispersal ability, propensity, and their interaction will provide insights into the mechanistic underpinnings of dispersal.

\section{Phenotypes of Immigrants versus Residents}

The natal conditions of one environment may affect neighboring populations through the phenotypes of the individuals that disperse into those populations; immigrants may differ from residents in fecundity, survival, or other fitness characteristics. Many examples exist of how natal habitats affect such relevant phenotypes of individuals in later life stages. For instance, buttercups (Ranunculus adoneus) in early snowmelt habitats produce higher-quality seeds that have greater fitness than buttercups from late snowmelt areas (Stanton et al. 1997). After metamorphosis, frogs that had developed as tadpoles with predators may differ in the length of their limbs (Relyea 2001; Van Buskirk and Saxer 2001) or in toxicity (Benard and Fordyce 2003) compared with those that had developed in the absence of predators. The dispersing phenotypes in wing dimorphic insects generally have reduced fecundity relative to the resident phenotypes because of a physiological trade-off between flight capacity and ovary mass (Zera and Denno 1997; Roff and Fairbairn 2001). By altering disperser phenotypes, natal habitats can have ecological and evolutionary effects that range from affecting the per capita recruitment rate of populations to altering the per capita effect of migrants on realized gene flow between populations. These ecological and evolutionary consequences have rarely been empirically investigated, but we discuss a few examples below.

\section{Implications of Natal Habitat-Induced Phenotypic Plasticity}

\section{Ecological Implications}

When the habitats that juveniles experience during development affect their ability and propensity to disperse away from the natal site, the dynamics and persistence of spatially structured populations and communities may critically differ from situations in which the proportion of dispersers is constant across all environments. For example, this may occur when natal habitat effects create an asymmetry in the dispersal rate between the two environments. Most metapopulation models assume symmetrical dispersal between patches (Levins 1969; Hanski 1999). Models that do incorporate asymmetry in the dispersal of individuals between patches have found that asymmetries strongly influence the conditions under which the entire system will persist (Saether et al. 1999; Amarasekare 2004;
Vuilleumier and Possingham 2006). Vuilleumier and Possingham (2006) explicitly modeled the consequences of dispersal asymmetries in metapopulations. They found that in comparison to models of symmetrical betweenpatch dispersal, asymmetrical dispersal increased the risk for whole population extinction. Further, more patches were required under conditions of asymmetric dispersal for the regional population to persist. Numerous factors can affect a patch's net number of emigrants; the most commonly considered examples are population size (or proxies of population size, including habitat area [Hanski 1999] or habitat quality [Moilanen and Hanski 2006]) and conspecific density (Saether et al. 1999; Amarasekare 2004). However, the empirical studies reviewed above demonstrate that the rate of emigration from a patch is also likely to be affected by how natal habitat affects adult dispersal phenotypes. Identification of how natal conditions affect dispersal propensity in a given taxa will allow ecologists to specifically test whether environmentally induced between-habitat asymmetries in dispersal affect metapopulations, as predicted by theory. For example, species that experience greater between-patch heterogeneity in the effects of natal habitat on dispersal would be predicted to have greater frequencies of extinction or be more susceptible to habitat fragmentation (Vuilleumier and Possingham 2006).

Habitat connectivity will also be affected by patch-level asymmetries in dispersal behavior, including dispersal rate and dispersal distance. Functional connectivity, the extent of movement between patches based on landscape configuration and dispersal behavior, critically affects patch occupancy, patch dynamics, and long-term persistence in spatially subdivided populations (Hanski 1999; Crooks and Sanjayan 2006). Natal habitat effects on individual dispersal probability, and thus net population emigration rate, have implications for the extent of regional connectivity between patches. Standard measures of habitat connectivity incorporate patch population size either directly or through surrogates including patch area and patch quality (Moilanen and Nieminen 2002; Moilanen and Hanski 2006). Models of population processes using standard connectivity metrics have had mixed success in predicting aspects of population movement between habitats, which has led some to argue that including more biological detail will improve these models' predictive ability (Winfree et al. 2005). Connectivity metrics typically assume that population size alone predicts the number of individuals a patch will contribute to the regional pool of dispersers, that is, that dispersers are a constant fraction of the local population. Yet this assumption is rarely likely to be true in nature. Evidence from some systems indicates that the proportion of dispersers from a given habitat may be inversely related to conspecific density or affected by dif- 
ferences in habitat condition so that individuals disperse to and away from sites proportionally to achieve an ideal free distribution (Doncaster et al. 1997; Diffendorfer 1998; Haugen et al. 2006). Our review suggests that patch environment can affect the development of connectivity between local sites by generating repeatable differences between types of patches and the probability that individuals leave their natal patch. Therefore, models of habitat connectivity should incorporate a term that reflects the proportion of likely dispersers as well as the absolute size of the patch when calculating the patch's contribution to the dispersal pool. This may lead to substantially different estimates of habitat connectivity than population size or area alone.

In addition to affecting individual dispersal probabilities, natal habitat conditions may affect the distance a dispersing individual travels. While dispersal distance is a critical component in estimating connectivity among habitat patches, most studies calculate connectivity using a single average dispersal distance applied to all patches. Estimates of dispersal distance are often derived from mark-release-recapture (MRR) data (Hanski 1999) or from radio-tracking data (e.g., Ozgul et al. 2006) collected on individuals within the landscape for which connectivity is calculated. This value is then applied to all patches in calculating their contribution to connectivity (Hanski 1999). This approach ignores differences in the distance traveled by dispersing individuals from patches with different natal conditions. When applied globally to all patches within a population, variation in average dispersal distance values has little effect on the relative connectivity of patches; that is, changing the value of average dispersal distance does not change the rank order of patch connectivity (Hanski 1999; Moilanen and Nieminen 2002). However, applying dispersal distance values that are specific to the different patch types can change both the absolute and the relative connectivity of a group of habitat patches. This has important implications for how connectivity is measured in natural populations.

Consider a hypothetical example in which dispersal distance estimates may be based on measurements of individuals from a single habitat type, even though individuals from other habitats may differ in average dispersal distance. This could occur for a number of reasons, for example, if sampling individuals from one patch type is easier than sampling individuals from other patch types. If the sampled individuals disperse for longer distances than individuals from the unsampled patches, the researchers will overestimate average patch connectivity. In turn, this would overestimate metapopulation capacity (Moilanen and Hanski 2006), which strongly affects the regional extinction threshold of a species (Ovaskainen and Hanski 2003). Therefore, overestimating the dispersal distance of individuals from some patches within a landscape can underestimate the risk of extinction to the whole regional population. This approach to estimating average dispersal distance also fails to correctly predict spatial variation in connectivity, autocorrelation between quality and connectivity, and the relative value of the patches to landscape connectivity. Because these factors can affect the time a patch takes to recover following disturbance and decrease the probability that a good-quality patch will be recolonized if it becomes locally extinct, incorrect estimates of connectivity negatively affect our conservation decisions. Further, increased variation in patch connectivity and changes in rank order also affect the relative value of conserving a given patch and its contribution to maintaining whole-landscape connectivity. Therefore, we feel that a better approach to this problem is to estimate average dispersal distance based on MRR or tracking data from multiple patch types. These are best-case conditions for a well-studied population in which measures of dispersal distance are made on individuals from enough patches that the range of habitat quality the population encounters and the effect of habitat quality on dispersal distance can be explicitly incorporated. Including dispersal distance parameterized to the specific patch type will provide substantially better estimates of how each population contributes to regional connectivity. Ultimately, recognizing and incorporating natal habitat effects into estimates of connectivity will improve our ability to predict and manage habitat connectivity.

Integrating the effects of habitat quality on dispersal distance does not, however, need to lead to irreducibly complex scenarios and individual patch-based average dispersal distance values. Empirical dispersal data are difficult to obtain, and we do not suggest that estimates of average dispersal distance values need be parameterized to each patch in a landscape. Instead, a description of the general relationships between habitat quality and dispersal distance (e.g., by understanding the relationships between habitat condition and body size and between body size and movement capacity) can produce reasonable patchtype estimates of average dispersal distance for many systems, which can then be incorporated into connectivity measures. Data on dispersal distance for individuals from different patches already exist in many MRR or tracking studies, and exploring the effects of applying patch-type dispersal distances on connectivity should begin here. There is an awareness in the ecological community of the importance of integrating the context (matrix or landscape) in which patches are located to understand habitat connectivity and factors affecting interhabitat dispersal (Wiens 2001; Moilanen and Hanski 2006). This remains an important area of work, critical to understanding the spatial connectivity of habitats. However, just as not all 
matrix environments are the same, neither are all habitat patches; both levels of environmental variation need to be integrated to effectively study spatial ecology and measure connectivity.

Ecological preferences may also be affected by natal habitat if and when natal habitat preference induction occurs (Davis and Stamps 2004). Preference for natal habitat conditions should decrease the exchange of individuals between sites that differ in those conditions, and dispersing individuals will perceive fewer habitat patches as potentially suitable sites for settlement. However, the effects of natal habitat preference induction on metapopulation dynamics have not been explored either theoretically or empirically. The effect of natal habitat preference induction on metapopulation dynamics will depend on several factors that include the strength of the preference, how habitat selection preferences change over increasing search distances, and the relative frequency of each habitat type. Further work on natal habitat preference induction and its consequences for population dynamics will lead to new insights on how local conditions can shape the regional connectivity of populations.

Natal habitat effects can influence ecological processes not only by altering the number or symmetry of dispersing individuals between two environments but also by altering the distribution of phenotypes within an environment. A population's phenotype distribution can strongly affect population-level ecological traits, such as population growth rate or carrying capacity (Bolnick et al. 2003). For example, the composition of male behavioral phenotypes (individuals scored as passive to aggressive) in experimental populations of water striders (Aquarius remigis) has a strong effect on female mating behavior; groups of very aggressive males actually drive females out of the population, thus potentially reducing the population-level reproductive output (Sih and Watters 2005). Similarly, the phenotypic mix within a population affected ecosystemlevel processes in plant communities (Madritch and Hunter 2004). If dispersers differ in phenotype from individuals that remain at the natal site, the impacts on the populations and communities that receive these dispersers may be different than predicted based on assuming the impact of one individual of the average phenotype to the site. Thus, in evaluating the effects of natal habitat-induced phenotypic plasticity on dispersal itself, researchers must also consider the impact of the phenotypes of dispersers. A useful line of future research will explore how the phenotypic mix affects population-, community-, and even ecosystem-level processes within donor and receiver patches.
Genetic Differentiation, Local Adaptation, and Speciation

Natal environmental conditions can affect the rate and symmetry of gene flow between populations. Environmental effects that increase the numbers of dispersers between populations will increase gene flow between those populations, assuming other factors are equal. Less obviously but potentially as important, natal environments may alter gene flow between populations by affecting the phenotypes of dispersers. For instance, even in the absence of asymmetries in dispersal rate, if individuals that originate from a high-quality habitat produce more offspring than individuals from a low-quality habitat, effective gene flow will be higher from the high-quality to the low-quality habitat than that predicted by numbers of dispersers alone. High gene flow between populations in different environments can limit adaptation to local conditions by overwhelming locally adapted genotypes with maladapted immigrant genotypes (Slatkin 1987). By limiting local adaptation, gene flow can not only inhibit speciation (Slatkin 1987) but also profoundly affect the ability of a species to persist in a habitat (e.g., Holt and Gomulkiewicz 1997; Ronce and Kirkpatrick 2001) and the ecological interactions among species (Travis 1996; Urban and Skelly 2006). A growing number of empirical examples demonstrate the occurrence of gene flow as a limiting factor for local adaptation in nature, highlighting its general importance (Stanton and Galen 1997; Storfer and Sih 1998; Hendry et al. 2002). Yet few empirical or theoretical investigations have considered whether environmentally induced differences in the capacity or motivation to disperse affect the frequency or degree of local adaptation that we expect to find in nature.

Many models of selection-gene flow balance consider a constant migration rate along a cline or between populations (Slatkin 1973; Garcia-Ramos and Kirkpatrick 1997). Under these cases, the models predict a characteristic distance over which the effect of dispersal (gene flow) on allele frequencies is greater than the effect of selection, resulting in limited local adaptation. Other models explicitly incorporate the migration of individuals between populations as the product of a constant migration rate and a variable population size (Holt and Gaines 1992; Hendry et al. 2001; Ronce and Kirkpatrick 2001). Natal habitat-induced changes in dispersal rate can affect genetic differentiation between populations in two ways: by altering the magnitude of gene flow between populations and by altering the symmetry of gene flow between populations. In the first case, habitat selection behavior shaped by natal experience (e.g., if individuals have a learned aversion or preference for the habitat in which they develop; Davis and Stamps 2004) can increase or decrease the rate at which local adaptation and speciation occur by altering 
the amount of realized gene flow (Beltman and Metz 2005). The second case, in which natal habitat effects generate an asymmetry in dispersal between populations, alters a common assumption of many models of the evolution of phenotypic plasticity and local adaptation in the face of gene flow.

If the assumption of equal migration rates across all environments is violated, do the predictions of models still hold? Models of the evolution of adaptive phenotypic plasticity in spatially variable environments have considered dispersal to be uncorrelated with phenotype or natal environment (de Jong 1999; Sultan and Spencer 2002). Yet, as described above, adaptive phenotypic plasticity in one trait or one life stage may affect dispersal capacity and motivation and, thus, patterns of dispersal and potentially the predictions of theory about the evolution of plasticity in response to local conditions. Environmentally induced asymmetry in dispersal has been investigated in models of gene flow-selection balance. In an appendix to the article by Stanton et al. (1997), Turelli showed that environmentally induced differences in offspring quality can substantially affect the magnitude of an asymmetry in realized gene flow across two habitat patches. Under these conditions, the allele frequencies of propagules from higherquality environments can swamp the frequency of alleles from lower-quality environments in both habitats. Kawecki and Holt (2002) evaluated the effect of asymmetry in dispersal rate between two patches on the probability of fixation of alleles that were advantageous in one patch but disadvantageous in another. Under two different sets of genetic assumptions, they found that local adaptation was more likely to occur in the population with a higher emigration rate. However, their predictions were also dependent on the ecological effects of immigration (e.g., density-dependent expression of fitness differences between genotypes). These theoretical studies demonstrate that environmentally induced asymmetries in the number or quality of dispersing individuals can lead to asymmetry in patterns of local adaptation.

Most empirical work on local adaptation has not investigated the consequences of asymmetries in dispersal leading to asymmetries in local adaptation. Instead, empirical work on the interplay between local adaptation and gene flow has generally used estimates of gene flow that cannot detect asymmetries (e.g., $\mathrm{F}_{\text {st }}$ as a surrogate for gene flow; Smith et al. 1997; Storfer and Sih 1998). However, a few studies demonstrate the importance of considering habitat-specific, nongenetic factors that create asymmetry in gene flow between habitats. Stanton et al. (1997) found that environmentally induced differences in offspring quality along a snowmelt gradient caused an asymmetrical pattern of gene flow from early-melt areas to late-melt areas over approximately $100 \mathrm{~m}$. The asymmetry in gene flow may have prevented adaptive genetic differentiation of the late-melt areas (Stanton and Galen 1997). This study demonstrates that such nongenetic, environmentally induced effects can have large, detectable consequences for gene flow across small spatial scales. In an example with animals, Garant et al. (2005) studied a population of great tits (Parus major) found across two habitats. An interaction between habitat quality and individual quality affected individual bird's dispersal decisions, so that high-quality birds settled in the higher-quality habitat. Over 19 generations, these habitat-induced differences in dispersal resulted in two distinct patterns of genetic adaptation and phenotypic change in the patches. These studies demonstrate the potential breadth of the role of environmentally induced asymmetry in dispersal between environments and its consequences for genetic differentiation and local adaptation. By affecting these processes, environmentally induced dispersal phenotypes may ultimately affect processes ranging from speciation to ecological interactions.

Evaluating how organisms' natal environments affect dispersal phenotypes and thus the magnitude and asymmetry of gene flow between environments will provide an opportunity to make a priori and even quantitative predictions about the expected magnitude of genetic differentiation and local adaptation. However, asymmetry in local adaptation provides potential pitfalls for researchers; habitat-induced asymmetry in dispersal may lead to completely asymmetrical adaptation, so that populations in two environments do not differ in genetic composition, but one is locally adapted and the other is maladapted (Ronce and Kirkpatrick 2001; Kawecki and Holt 2002). Under these conditions, standard tests for local adaptation, such as reciprocal transplant experiments or common garden experiments, would be unable to discriminate this from a failure of both populations to become locally adapted, because the populations are not genetically differentiated. Thus, researchers should combine either reciprocal transplant or common garden experiments with estimates of the fitness landscape in different environments; under the scenario of complete asymmetry in local adaptation, fitness landscapes would reveal directional selection in the completely maladapted population but stabilizing selection in the completely locally adapted population. Estimation of fitness surfaces in addition to reciprocal transplant experiments in different habitats would reveal this potential problem but is rarely used (but see Bennington and McGraw 1995).

\section{Conclusions and Future Research Directions}

Environmentally induced asymmetry in the numbers or phenotypes of dispersers is likely to be widespread and can have substantial effects on both ecological and evolutionary 
processes. We envision a research program investigating these effects to operate at three levels. First, the environmental conditions that affect the phenotypes must be established; for example, environments with lower predation risk cause individuals to have a higher growth rate and thus attain a larger size at metamorphosis. Second, the mechanism by which natal habitat-induced variation in phenotypic traits affects dispersal must be identified. How natal habitats affect dispersal will depend on whether dispersal is governed by decision rules that maximize fitness or whether it is a by-product of other adaptive behaviors (e.g., foraging) in a nonreproductive life stage. Third, the ecological and evolutionary consequences of environmentally induced variation in the numbers or phenotypes of dispersers must be identified (e.g., has environmentally induced asymmetry in gene flow generated landscape-level patterns of asymmetry in local adaptation?).

A critical component of this research program is to assess the temporal and spatial scale of variation in these environmental traits. Researchers must distinguish between environmental characteristics that affect dispersal and differ between populations in a consistent, repeatable manner over time and environmental traits that affect dispersal but exhibit no correlation with geography. Natal environments may have a strong effect on dispersal phenotypes, but if environmental variation among patches is entirely temporal and not spatial, the consequences for populations may be qualitatively different from those described here. However, patchy spatial structure among heterogeneous environmental conditions is widespread in nature, including differences in host-plant distributions for phytophagous insects (Jaenike 1990), soil type distributions for plants (e.g., Brady et al. 2005), and characteristics of freshwater ponds for many insects and amphibians (Wellborn et al. 1996). Thus, there are many spatially variable environmental conditions that may create consistent differences in disperser phenotypes.

Relating the dispersal stage phenotype to actual dispersal can be accomplished on multiple levels that together integrate individual-scale functional morphology and behavior with observed patterns of dispersal and gene flow. Laboratory trials can compare dispersal ability (e.g., Beck and Congdon 2000) and motivation (e.g., Coll and Yuval 2004) in individuals reared in, or collected from, varying natal conditions. Direct estimates of dispersal can be used to evaluate the importance of individual phenotype, dispersal capacity, and motivation for realized dispersal between populations (e.g., Garant et al. 2005). Indirect estimates of the degree of asymmetry in gene flow between different environmental conditions can be estimated with a growing number of statistical approaches using molecular genetic data (e.g., Pearse and Crandall 2004). Comparison of direct and indirect estimates of differences in dispersal between habitats allows a valuable cross-validation of both approaches. These varied approaches can all provide critical information for evaluating patterns of environmentally induced asymmetry in dispersal.

The primary goal of this research program is to identify how these environmentally induced changes in the numbers and phenotypes of dispersers affect ecological and evolutionary processes. Once the linkages of environment to phenotype and phenotype to actual dispersal have been made, researchers can then investigate their impact. We predict that these impacts will be substantial, ranging from altering the patterns and magnitude of connectivity and local adaptation to changing the abundance of a species on the landscape. Multiple comparative and experimental approaches can be used to investigate these impacts. Comparative studies on multiple species or sets of populations can test predictions for how the magnitude of natal habitat effects on dispersal affects the degree of local adaptation or patch occupancy. Connectivity values scaled by patch environment-specific dispersal rates or distances can be compared with simpler connectivity measures to determine whether they more accurately predict extinction and colonization. If so, this suggests an important modification of how habitats are selected for conservation purposes. Experimental studies that manipulate how natal habitats affect dispersal can be conducted in microcosms or on larger scales. For instance, separate landscapes of host plants or pond environments that affect insect or amphibian dispersal capacity can be created. Together, comparative and experimental approaches can reveal the details of how natal habitat effects translate from the individual level to the population level. Ultimately, understanding the role of natal habitat effects on dispersal and disperser phenotypes will provide a deeper insight into the interaction of local and regional processes in ecology and evolution.

\section{Acknowledgments}

M.F.B. was supported by the Michigan Society of Fellows and the University of Michigan Department of Ecology and Evolutionary Biology. S.J.M. was supported by the University of California Davis Center for Population Biology and worked on this manuscript while in the laboratory of S. Lawler in the University of California Davis Department of Entomology. This manuscript was improved by comments from B. Ajie, J. Clobert, C. Cressler, C. Davis, E. Farrer, S. Fogarty, M. Fraker, J. Hammond, T. Iglesias, E. Kisdi, B. Luttbeg, J. Middlemis-Brown, L. Pintor, J. Saltz, S. Schneider, A. Sih, H. Watts, E. Werner, W. White, and A. Zellmer.

\section{Literature Cited}

Amarasekare, P. 2004. The role of density-dependent dispersal in source-sink dynamics. Journal of Theoretical Biology 226:159-168. 
Angelibert, S., and N. Giani. 2003. Dispersal characteristics of three odonate species in a patchy habitat. Ecography 26:13-20.

Anholt, B. R. 1990. Size-biased dispersal prior to breeding in a damselfly. Oecologia (Berlin) 83:385-387.

Baker, M. B., and S. Rao. 2004. Incremental costs and benefits shape natal dispersal: theory and example with Hemilepistus reaumuri. Ecology 85:1039-1051.

Barbraud, C., A. R. Johnson, and G. Bertault. 2003. Phenotypic correlates of post-fledging dispersal in a population of greater flamingos: the importance of body condition. Journal of Animal Ecology 72:246-257.

Beck, C. W., and J. D. Congdon. 2000. Effects of age and size at metamorphosis on performance and metabolic rates of southern toad, Bufo terrestris, metamorphs. Functional Ecology 14:32-38.

Bélichon, S., J. Clobert, and M. Massot. 1996. Are there differences in fitness components between philopatric and dispersing individuals? Acta Oecologica 17:503-517.

Beltman, J. B., and J. A. J. Metz. 2005. Speciation: more likely through a genetic or through a learned habitat preference? Proceedings of the Royal Society B: Biological Sciences 272:1455-1463.

Benard, M. F. 2004. Predator-induced phenotypic plasticity in organisms with complex life histories. Annual Review of Ecology Evolution and Systematics 35:651-673.

Benard, M. F., and J. A. Fordyce. 2003. Are induced defenses costly? consequences of predator-induced defenses in western toads, Bufo boreas. Ecology 84:68-78.

Bengsston, B. O. 1978. Avoiding inbreeding: at what cost? Journal of Theoretical Biology 73:439-444.

Bennington, C. C., and J. B. McGraw. 1995. Natural selection and ecotypic differentiation in Impatiens pallida. Ecological Monographs 65:303-323.

Blanckenhorn, W. U. 2006. Divergent juvenile growth and development mediated by food limitation and foraging in the water strider Aquarius remigis (Heteroptera: Gerridae). Journal of Zoology 268:17-23.

Bolnick, D. I., R. Svanback, J. A. Fordyce, L. H. Yang, J. M. Davis, C. D. Hulsey, and M. L. Forister. 2003. The ecology of individuals: incidence and implications of individual specialization. American Naturalist 161:1-28.

Bonte, D., N. Vandenbroecke, L. Lens, and J. P. Maelfait. 2003. Low propensity for aerial dispersal in specialist spiders from fragmented landscapes. Proceedings of the Royal Society B: Biological Sciences 270:1601-1607.

Brady, K. U., A. R. Kruckeberg, and H. D. Bradshaw. 2005. Evolutionary ecology of plant adaptation to serpentine soils. Annual Review of Ecology Evolution and Systematics 36:243-266.

Clobert, J., E. Danchin, A. A. Dhondt, and J. D. Nichols. 2001. Dispersal. Oxford University Press, Oxford.

Clobert, J. E., R. A. Ims, and F. Rousset. 2004. Causes, mechanisms, and consequences of dispersal. Pages 307-335 in I. Hanksi and O. Gaggiotti, eds. Ecology, genetics and evolution of metapopulations. Academic Press, London.

Cohen, D., and U. Motro. 1989. More on optimal rates of dispersal: taking into account the cost of the dispersal mechanism. American Naturalist 134:659-663.

Coll, M., and B. Yuval. 2004. Larval food plants affect flight and reproduction in an oligophagous insect herbivore. Environmental Entomology 33:1471-1476.

Comins, H. N., W. D. Hamilton, and R. M. May. 1980. Evolutionary stable dispersal strategies. Journal of Theoretical Biology 82:205230.

Conrad, K. F., K. H. Willson, K. Whitfield, I. F. Harvey, C. J. Thomas, and T. N. Sherratt. 2002. Characteristics of dispersing Ischnura elegans and Coenagrion puella (Odonata): age, sex, size, morph and ectoparasitism. Ecography 25:439-445.

Crespi, B. J., and P. D. Taylor. 1990. Dispersal rates under variable patch density. American Naturalist 135:48-62.

Crooks, K. R., and M. Sanjayan. 2006. Connectivity conservation. Oxford University Press, New York.

Davis, J. M., and J. A. Stamps. 2004. The effect of natal experience on habitat preferences. Trends in Ecology \& Evolution 19:411-416.

de Fraipont, M., J. Clobert, H. John-Alder, and S. Meylan. 2000. Increased pre-natal maternal corticosterone promotes philopatry of offspring in common lizards Lacerta vivipara. Journal of Animal Ecology 69:404-413.

de Jong, G. 1999. Unpredictable selection in a structured population leads to local genetic differentiation in evolved reaction norms. Journal of Evolutionary Biology 12:839-851.

Denno, R. F., D. J. Hawthorne, B. L. Thorne, and C. Gratton. 2001. Reduced flight capability in British Virgin Island populations of a wing-dimorphic insect: the role of habitat isolation, persistence, and structure. Ecological Entomology 26:25-36.

Diffendorfer, J. E. 1998. Testing models of source-sink dynamics and balanced dispersal. Oikos 81:417-433.

Dingle, H. 1996. Migration: the biology of life on the move. Oxford University Press, New York.

Doebeli, M., and G. D. Ruxton. 1997. Evolution of dispersal rates in metapopulation models: branching and cyclic dynamics in phenotype space. Evolution 51:1730-1741.

Doncaster, C. P., J. Clobert, B. Doligez, L. Gustafsson, and E. Danchin. 1997. Balanced dispersal between spatially varying local populations: an alternative to the source-sink model. American Naturalist 150:425-445.

Dudley, R. 1990. Biomechanics of flight in Neotropical butterflies: morphometrics and kinematics. Journal of Experimental Biology 150:37-53.

-2000. The biomechanics of insect flight. Princeton University Press, Princeton, NJ.

Dufty, A. M., Jr., J. Clobert, and A. P. Møller. 2002. Hormones, developmental plasticity, and adaptation. Trends in Ecology \& Evolution 17:190-196.

Garant, D., L. E. B. Kruuk, T. A. Wilkin, R. H. McCleery, and B. C. Sheldon. 2005. Evolution driven by differential dispersal within a wild bird population. Nature 433:60-65.

Garcia-Ramos, G., and M. Kirkpatrick. 1997. Genetic models of adaptation and gene flow in peripheral populations. Evolution 51: 21-28.

Hamilton, W. D., and R. M. May. 1977. Dispersal in stable habitats. Nature 269:578-581.

Hanski, I. 1991. Natal dispersal and social dominance in the common shrew Sorex araneus. Oikos 62:48-58.

1999. Metapopulation ecology. Oxford University Press, Oxford.

Hanski, I., and M. E. Gilpin. 1997. Metapopulation biology: ecology, genetics, and evolution. Academic Press, San Diego, CA.

Haugen, T. O., I. J. Winfield, L. A. Vollestad, J. M. Fletcher, J. B. James, and N. C. Stenseth. 2006. The ideal free pike: 50 years of fitness-maximizing dispersal in Windermere. Proceedings of the Royal Society B: Biological Sciences 273:2917-2924. 
Hayward, L. S., and J. C. Wingfield. 2004. Maternal corticosterone is transferred to avian yolk and may alter offspring growth and adult phenotype. General and Comparative Endocrinology 135: 365-371.

Hendry, A. P., T. Day, and E. B. Taylor. 2001. Population mixing and the adaptive divergence of quantitative traits in discrete populations: a theoretical framework for empirical tests. Evolution 55: 459-466.

Hendry, A. P., E. B. Taylor, and J. D. McPhail. 2002. Adaptive divergence and the balance between selection and gene flow: lake and stream stickleback in the misty system. Evolution 56:11991216.

Hockey, P. A. R., A. Leseberg, and D. Lowenthal. 2003. Dispersal and migration of juvenile African black oystercatchers Haematopus moquini. Ibis 145:E114-E123.

Holekamp, K. E. 1984. Natal dispersal in Belding's ground squirrels (Spermophilus beldingi). Behavioral Ecology and Sociobiology 16: 21-30.

- 1986. Proximal causes of natal dispersal in Belding's ground squirrels (Spermophilus beldingi). Ecological Monographs 56:365391.

Holt, R. D., and M. S. Gaines. 1992. Analysis of adaptation in heterogeneous landscapes: implications for the evolution of fundamental niches. Evolutionary Ecology 6:433-447.

Holt, R. D., and R. Gomulkiewicz. 1997. How does immigration influence local adaptation? a reexamination of a familiar paradigm. American Naturalist 149:563-572.

Holt, R. D., and M. A. McPeek. 1996. Chaotic population dynamics favors the evolution of dispersal. American Naturalist 148:709718.

Holyoak, M., M. A. Leibold, and R. D. Holt. 2005. Metacommunities: spatial dynamics and ecological communities. University of Chicago Press, Chicago.

Imbert, E., and O. Ronce. 2001. Phenotypic plasticity for dispersal ability in the seed heteromorphic Crepis sancta (Asteraceae). Oikos 93:126-134.

Jaenike, J. 1990. Host specialization in phytophagous insects. Annual Review of Ecology and Systematics 21:243-273.

Johnson, M. L., and M. S. Gaines. 1990. Evolution of dispersal: theoretical models and empirical tests using birds and mammals. Annual Review of Ecology and Systematics 21:449-480.

Kawecki, T. J., and R. D. Holt. 2002. Evolutionary consequences of asymmetric dispersal rates. American Naturalist 160:333-347.

Kisdi, E. 2002. Dispersal: risk spreading versus local adaptation. American Naturalist 159:579-596.

Lens, L., and A. A. Dhondt. 1994. Effects of habitat fragmentation on the timing of crested tit Parus cristatus natal dispersal. Ibis 136: 147-152.

Levin, S. A., D. Cohen, and A. Hastings. 1984. Dispersal strategies in patchy environments. Theoretical Population Biology 26:165-191.

Levins, R. 1969. Some demographic and genetic consequences of environmental heterogeneity for biological control. Bulletin of the Entomological Society of America 15:237-240.

Madritch, M. D., and M. D. Hunter. 2004. Phenotypic diversity and litter chemistry affect nutrient dynamics during litter decomposition in a two species mix. Oikos 105:125-131.

Marchlewska-Koj, A., J. Kapusta, and M. Kruczek. 2003. Prenatal stress modifies behavior in offspring of bank voles (Clethrionomys glareolus). Physiology \& Behavior 79:671-678.

Massot, M., and J. Clobert. 1995. Influence of maternal food avail- ability on offspring dispersal. Behavioral Ecology and Sociobiology 37:413-418.

Mathias, A., E. Kisdi, and I. Olivieri. 2001. Divergent evolution of dispersal in a heterogeneous landscape. Evolution 55:246-259.

McPeek, M. A., and R. D. Holt. 1992. The evolution of dispersal in spatially and temporally varying environments. American Naturalist 140:1010-1027.

Merckx, T., and H. Van Dyck. 2006. Landscape structure and phenotypic plasticity in flight morphology in the butterfly Pararge aegeria. Oikos 113:226-232.

Meylan, S., and J. Clobert. 2004. Maternal effects on offspring locomotion: influence of density and corticosterone elevation in the lizard Lacerta vivipara. Physiological and Biochemical Zoology 77: 450-458.

Meylan, S., J. Belliure, J. Clobert, and M. de Fraipont. 2002. Stress and body condition as prenatal and postnatal determinants of dispersal in the common lizard (Lacerta vivipara). Hormones and Behavior 42:319-326.

Meylan, S., M. De Fraipont, and J. Clobert. 2004. Maternal size and stress and offspring philopatry: an experimental study in the common lizard (Lacerta vivipara). Ecoscience 11:123-129.

Michiels, N. K., and A. A. Dhondt. 1991. Characteristics of dispersal in sexually mature dragonflies. Ecological Entomology 16:449-459.

Moilanen, A., and I. Hanski. 2006. Connectivity and metapopulation dynamics in highly fragmented landscapes. Pages $44-71$ in K. R. Crooks and M. Sanjayan, eds. Connectivity conservation. Cambridge University Press, New York.

Moilanen, A., and M. Nieminen. 2002. Simple connectivity measures in spatial ecology. Ecology 83:1131-1145.

Morey, S., and D. Reznick. 2000. A comparative analysis of plasticity in larval development in three species of spadefoot toads. Ecology 81:1736-1749.

Nieminen, M., H. Rita, and P. Uuvana. 1999. Body size and migration rate in moths. Ecography 22:697-707.

Nosil, P. and B. J. Crespi. 2004. Does gene flow constrain adaptive divergence or vice versa? a test using ecomorphology and sexual isolation in Timema cristinae walking-sticks. Evolution 58:102-112.

Nunes, S., C. D. T. Ha, P. J. Garrett, E. M. Mueke, L. Smale, and K. E. Holekamp. 1998. Body fat and time of year interact to mediate dispersal behaviour in ground squirrels. Animal Behaviour 55:605614

O’Riain, J. M., J. U. M. Jarvis, and C. G. Faulkes. 1996. A dispersive morph in the naked mole-rat. Nature 380:619-621.

Ovaskainen, O., and I. Hanski. 2003. Extinction threshold in metapopulation models. Annales Zoologici Fennici 40:81-97.

Ozgul, A., K. B. Armitage, D. T. Blumstein, D. H. Vanvuren, and M. K. Oli. 2006. Effects of patch quality and network structure on patch occupancy dynamics of a yellow-bellied marmot metapopulation. Journal of Animal Ecology 75:191-202.

Paradis, E., S. R. Baillie, W. J. Sutherland, and R. D. Gregory. 1998. Patterns of natal and breeding dispersal in birds. Journal of Animal Ecology 67:518-536.

Pearse, D. E., and K. A. Crandall. 2004. Beyond $\mathrm{F}_{\mathrm{ST}}$ : analysis of population genetic data for conservation. Conservation Genetics 5:585-602.

Pechenik, J. A. 2006. Larval experience and latent effects: metamorphosis is not a new beginning. Integrative and Comparative Biology 46:323-333.

Relyea, R. A. 2001. The lasting effects of adaptive plasticity: predatorinduced tadpoles become long-legged frogs. Ecology 82:1947-1955. 
Roach, D. A., and R. D. Wulff. 1987. Maternal effects in plants. Annual Review of Ecology and Systematics 18:209-235.

Roff, D. A. 1991. Life history consequences of bioenergetic and biomechanical constraints on migration. American Zoologist 31:243251.

Roff, D. A., and D. J. Fairbairn. 1991. Wing dimorphisms and the evolution of migratory polymorphisms among the Insecta. American Zoologist 31:243-251.

- 2001. The genetic basis of dispersal and migration, and its consequences for the evolution of correlated traits. Pages 191-202 in J. Clobert, E. Danchin, A. A. Dhondt, and J. D. Nichols, eds. Dispersal. Oxford University Press, Oxford.

Ronce, O., and M. Kirkpatrick. 2001. When sources become sinks: migrational meltdown in heterogeneous habitats. Evolution 55: 1520-1531.

Ronce, O., I. Olivieri, J. Clobert, and E. Danchin. 2001. Perspectives on the study of dispersal evolution. Pages 341-357 in J. Clobert, E. Danchin, A. A. Dhondt, and J. D. Nichols. 2001. Dispersal. Oxford University Press, Oxford.

Roslin, T. 2000. Dung beetle movements at two spatial scales. Oikos 91:323-335.

Rossi, A. M., B. V. Brodbeck, and D. R. Strong. 1996. Response of xylem-feeding leafhopper to host plant species and plant quality. Journal of Chemical Ecology 22:653-671.

Saether, B. E., S. Engen, and R. Lande. 1999. Finite metapopulation models with density-dependent migration and stochastic local dynamics. Proceedings of the Royal Society B: Biological Sciences 266:113-118

Schilder, R. J., and J. H. Marden. 2004. A hierarchical analysis of the scaling force and power production by dragonfly motors. Journal of Experimental Biology 207:767-776.

Shapiro, B. J., D. Garant, T. A. Wilkin, and B. C. Sheldon. 2006. An experimental test of the causes of small-scale phenotypic differentiation in a population of great tits. Journal of Evolutionary Biology 19:176-183.

Sih, A., and J. V. Watters. 2005. The mix matters: behavioural types and group dynamics in water striders. Behaviour 142:1417-1431.

Sinervo, B., R. Calsbeek, T. Comendant, C. Both, C. Adamopoulou, and J. Clobert. 2006. Genetic and maternal determinants of effective dispersal: the effect of sire genotype and size at birth in side-blotched lizards. American Naturalist 168:88-99.

Slatkin, M. 1973. Gene flow and selection in a cline. Genetics 75: 733-756.

. 1987. Gene flow and the geographic structure of natural populations. Science 236:787-792.

Sloggett, J. J., and W. W. Weisser. 2002. Parasitoids induce production of the dispersal morph of the pea aphid, Acyrthosiphon pisum. Oikos 98:323-333.

Smith, T. B., R. K. Wayne, D. J. Girman, and M. W. Bruford. 1997. A role for ecotones in generating rainforest biodiversity. Science 276:1855-1857.

Srygley, R. B., and P. Chai. 1990. Flight morphology of Neotropical butterflies: palatability and distribution of mass to the thorax and abdomen. Oecologia (Berlin) 84:491-499.

Stanton, M. L., and C. Galen. 1997. Life on the edge: adaptation versus environmentally mediated gene flow in the snow buttercup, Ranunculus adoneus. American Naturalist 150:143-178.
Stanton, M. L., C. Galen, and J. Shore. 1997. Population structure along a steep environmental gradient: consequences of flowering time and habitat variation in the snow buttercup, Ranunculus adoneus. Evolution 51:79-94.

Storfer, A., and A. Sih. 1998. Gene flow and ineffective antipredator behavior in a stream-breeding salamander. Evolution 52:558-565.

Sultan, S. E., and H. G. Spencer. 2002. Metapopulation structure favors plasticity over local adaptation. American Naturalist 160: 271-283.

Sutherland, G. D., A. S. Harestad, K. Price, and K. P. Lertzman. 2000. Scaling of natal dispersal distances in terrestrial birds and mammals. Conservation Ecology 4:16.

Travis, J. 1996. The significance of geographical variation in species interactions. American Naturalist 148(suppl.):S1-S8.

Urban, M. C., and D. K. Skelly. 2006. Evolving metacommunities: toward an evolutionary perspective on metacommunities. Ecology 87:1616-1626.

Van Buskirk, J., and G. Saxer. 2001. Delayed costs of an induced defense in tadpoles? morphology, hopping, and development rate at metamorphosis. Evolution 55:821-829.

van der Jeugd, H. P. 2001. Large barnacle goose males can overcome the social costs of natal dispersal. Behavioral Ecology 12:275-282.

van Oort, H., and K. A. Otter. 2005. Natal nutrition and the habitat distributions of male and female black-capped chickadees. Canadian Journal of Zoology 83:1495-1501.

Van Vuren, D., and K. B. Armitage. 1994. Survival of dispersing and philopatric yellow-bellied marmots: what is the cost of dispersal? Oikos 69:179-181.

Vercken, E., M. de Fraipont, A. M. Duffy Jr., and J. Clobert. 2007. Mother's timing and duration of corticosterone exposure modulate offspring size and natal dispersal in the common lizard (Lacerta vivipara). Hormones and Behavior 51:379-386.

Verhulst, S., C. M. Perrins, and R. Riddington. 1997. Natal dispersal of great tits in a patchy environment. Ecology 78:864-872.

Vuilleumier, S., and H. P. Possingham. 2006. Does colonization asymmetry matter in metapopulations? Proceedings of the Royal Society B: Biological Sciences 273:1637-1642.

Weisser, W. W., C. Braendle, and N. Minoretti. 1999. Predatorinduced morphological shift in the pea aphid. Proceedings of the Royal Society B: Biological Sciences 266:1175-1181.

Wellborn, G. A., D. K. Skelly, and E. E. Werner. 1996. Mechanisms creating community structure across a freshwater habitat gradient. Annual Review of Ecology and Systematics 27:337-363.

Whiteman, H. H. 1994. Evolution of facultative paedomorphosis in salamanders. Quarterly Review of Biology 69:205-221.

Wiens, J. A. 2001. The landscape context of dispersal. Pages 96-109 in J. Clobert, E. Danchin, A. A. Dhondt, and J. D. Nichols, eds. Dispersal. Oxford University Press, Oxford.

Winfree, R., J. Dushoff, E. E. Crone, C. B. Schultz, R. V. Budny, N. M. Williams, and C. Kremen. 2005. Testing simple indices of habitat proximity. American Naturalist 165:707-717.

Zera, A. J., and R. F. Denno. 1997. Physiology and ecology of dispersal polymorphism in insects. Annual Review of Entomology 42:207230.

Associate Editor: Nicolas Perrin Editor: Monica A. Geber 